Anaesthesist 2021 · 70:549-550

https://doi.org/10.1007/s00101-021-01004-8

Angenommen: 8. Juni 2021

(c) Springer Medizin Verlag $\mathrm{GmbH}$, ein Teil von Springer Nature 2021

\section{Cannabis - Es geht ums Ganze!}

Matthias Karst

Klinik für Anästhesiologie und Intensivmedizin, Schmerzambulanz, Medizinische Hochschule Hannover, Hannover, Deutschland
In dem Beitrag „Cannabis und Cannabinoide zur Therapie akuter und chronischer Schmerzen" in der aktuellen Ausgabe von Der Anaesthesist ist es den Autoren gelungen, ein sehr komplexes und in der Fachwelt kontrovers diskutiertes Thema sowohl auf der inhaltlichen als auch auf der regulatorischen Ebene umfassend und ausgewogen darzustellen [1].

Das sog. Cannabis-als-Medizin-Gesetz, das am 10.03.2017 in Kraft getreten ist, hat dazu geführt, dass cannabisbasierte Arzneimittel (CBA) auch bei chronischen Schmerzen verordnet werden können. Mit Ausnahme von Nabiximols und Nabilon handelt es sich um Rezepturarzneimittel. Die dabei gültige Arzneimittelpreisverordnung und der damit vorgeschriebene Preisaufschlag erhöhen die finanzielle Belastung der Kostenträger. So wundert es nicht, dass der im Gesetz verankerte Genehmigungsvorbehalt dazu führt, dass weiterhin in ca. $40 \%$ aller Fälle die Kostenübernahme abgelehnt wird, und nicht nur „in begründeten Ausnahmefällen“. Insbesondere das von den Kostenträgern angeführte Argument "fehlende Evidenz" stellt sich gegen die Intention des Gesetzgebers, der bewusst keine Indikation vorgegeben hat.

\section{॥ Zielkriterium muss die Verbes- serung des Schmerzaffekts und beeinträchtigter Lebensbereiche sein}

Dabei wird der Begriff „Evidenz" missbraucht, indem darunter ausschließlich Erkenntnisse aus datengestützter Medizin (randomisierte kontrollierte Studien, $\mathrm{RCT}$; Metaanalysen) verstanden werden und die Erfahrungswerte der Behandler und der Patienten unberücksichtigt blei- ben, woraus sich erst die evidenzbasierte Medizin (EBM) zusammensetzt. Dies gilt umso mehr, wenn es sich um ein so hoch subjektives Phänomen wie Schmerz handelt, der bekanntlich untrennbar mit der betroffenen Person verbunden ist und nicht mit einem objektiven Maß belegt werden kann. Während die Metaanalysen z.B. zu der Aussage gelangen, dass es unwahrscheinlich erscheint, dass Cannabinoide hochwirksame Medikamente für chronische Nichttumorschmerzen sind [2], weisen die Erfahrungen der Patienten in die entgegengesetzte Richtung [3]. Neben der außerordentlich hohen Heterogenität der RCT scheint v.a. die Auswahl der Outcome-Parameter die Studienergebnisse zu beeinflussen. Beispielhaft zeigt sich dies an einer prospektiven Kohortenstudie mit chronischen Schmerzpatienten, in die mehr als 1000 Patienten eingeschlossen waren [4]. Nach 12 Monaten Behandlung mit einem CBA ging die Schmerzintensität um 20\% zurück, die affektive Schmerzkomponente und Schlafstörung aber jeweils um $33 \%$, die Ausprägung von Depression und Angst um $32 \%$ bzw. $40 \%$ [4]. Es kam zu einem Einspareffekt von Morphinäquivalenten um $42 \%$ [4]. Deutlich wird hier, dass nicht ausschließlich die Reduktion der Schmerzintensität im Vordergrund steht, sondern eine Verbesserung in einer Vielzahl von relevanten Lebensbereichen, die mit chronischen Schmerzen assoziiert sind.

Bekanntlich entsteht chronischer Schmerz auf dem Boden einer Dysfunktion im kortikomesolimbischen System [5]. Erst kürzlich konnten Weizman et al. [6] bei Patienten mit chronischen Nervenschmerzen zeigen, dass der Effekt von $\Delta^{9}$-Tetrahydrocannabinol (THC) auf die Schmerzreduktion umso größer war, je stärker das dysfunktionale Konnektom im kortikomesolimbischen System ausgeprägt war [6]. Diese Beobachtungen deuten darauf hin, dass die Beeinflussung des Endocannabinoidsystems (ECS) im Gehirn, insbesondere die Aktivierung des Cannabinoidrezeptors CB1, für die Behandlung von chronischen Schmerzen von hoher Relevanz ist.

Um die mit der zentralen Aktivierung von CB1-Rezeptoren verbundenen cannabimimetischen Effekte zu vermeiden, wurden verschiedene Ansätze erprobt. Präklinische Daten zeigten, dass Analgesie durch ausschließliche Aktivierung von peripheren CB1-Rezeptoren erreicht werden kann [7]. In klinischen Untersuchungen konnte dies bisher nicht nachvollzogen werden. Auch die (indirekte) Erhöhung des Endocannabinoid(EC)-Tonus durch Einsatz von Inhibitoren der Fettsäureamidhydrolase (FAAH) oder der Monoacylglycerollipase (MAGL) konnte in klinischen Studien bislang nicht überzeugen [8]. Einzig die überwiegend an periphere $\mathrm{CB} 2-$ Rezeptoren bindende ajulämische Säure (AJA, [9]) zeigte in einer kleinen Studie eine mäßig ausgeprägte Schmerzreduktion bei Patienten mit chronischen Nervenschmerzen [10]. Diese Substanz befindet sich aktuell in der klinischen Entwicklung zur antiinflammatorischen Behandlung verschiedener Kollagenosen [11].

Solange Arzneimittel- und Zulassungsbehörden in "Schmerzstudien" auf einer Hierarchie der Zielparameter bestehen, mit Erfassung der Schmerzintensität mithilfe einer einfachen Skala als primärem Endpunkt, werden CBA es schwer haben, in RCT ihre klinische Bedeutung zu zeigen. Die Erfassung der affektiven Schmerzdimension und die Verwendung zusammengesetzter Scores, die die Schmerzerfah- 
rung und die Lebensqualität umfassend beschreiben, sind in einer solchen Situation geeigneter.

Unter Beteiligung der European Medicines Agency (EMA) wurden Wege aufgezeigt, wie in der klinisch-pharmazeutischen Forschung Nutzen-Risiko-Analysen durchgeführt werden können (Übersicht bei Nutt et al. [3]). Eine internationale Gruppe von Schmerztherapeuten mit und ohne Erfahrung in der Anwendung von CBA, von Psychiatern, Neurologen und Wissenschaftlern mit der Expertise in der Pharmakologie von Cannabinoiden sowie von Vertretern einer Patientengruppe (United Patient Alliance) hat eine solche Entscheidungsanalyse entwickelt [3]. Anhand von 17 nach klinischer Relevanz gewichteter Kriterien wurden 12 für chronische neuropathische Schmerzen häufig genutzte Arzneimittel bewertet, darunter CBA, Antidepressiva, Gabapentinoide und Opioide. Demnach scheinen CBA eine größere Bedeutung in Bezug auf die Verbesserung der Lebensqualität zu haben als auf die alleinige Reduktion der Schmerzintensität [3]. Dies gilt gerade im Vergleich zu Duloxetin, Gabapentinoiden und Amitriptylin [3]. Unter zusätzlicher Berücksichtigung des Nebenwirkungsprofils zeigten alle 3 CBA (1:1-Kombination von THC und Cannabidiol [CBD], THC, CBD) gegenüber allen anderen Substanzen einen Vorteil [3].

Die in Vollextrakten und Cannabisblüten enthaltenen Phytocannabinoide scheinen eine große Bedeutung in Bezug auf das Wirkungs-und Nebenwirkungsprofil zu haben. Patienten mit chronischen Schmerzen, die unter verschiedenen Cannabissorten auswählen konnten, haben sich nicht primär am Ausmaß der hervorgerufenen Schmerzreduktion orientiert, sondern an schmerzassoziierten Faktoren (z. B. Verbesserung des Nachtschlafs, [12]). Darüber hinaus zeigte sich, dass die Rate der Nebenwirkungen stark von dem Profil der Phytocannabinoide abhängt [12]. Folgerichtig ist zu empfehlen, Cannabissorten zu entwickeln, die sich durch ein wirksames und nebenwirkungsarmes Cannabinoid- und Terpenprofil auszeichnen. In Verbindung mit effektiver und sicherer Galenik kann erwartet werden, dass CBA das Armamentarium in der pharmakologischen Therapie chronischer Schmerzen erweitern werden.

\section{Korrespondenzadresse}

\section{Prof. Dr. Matthias Karst}

Klinik für Anästhesiologie und Intensivmedizin, Schmerzambulanz, Medizinische Hochschule Hannover

Carl-Neuberg-Str. 1, 30625 Hannover,

Deutschland

karst.matthias@mh-hannover.de

Interessenkonflikt. M. Karst ist Mitglied der Ad-hocKommission "Cannabis in der Medizin" der Deutschen Schmerzgesellschaft e.V.

\section{Literatur}

1. Schäfer M, Tafelski S (2021) Cannabis und Cannabinoide zur Therapie akuter und chronischer Schmerzen. Anaesthesist. https://doi.org/10. 1007/s00101-021-00994-9

2. Stockings E, Campbell G, Hall WD et al (2018) Cannabis and cannabinoids for the treatment of people with chronic noncancer pain conditions: a systematic review and meta-analysis of controlled and observational studies. Pain 159:1932-1954

3. Nutt DJ, Philipps LD, Barnes MP et al (2021) A multicriteria decision analysis comparing pharmacotherapy for chronic neuropathic pain, including cannabinoids and cannabis-based medical products. Cannabis Cannabinoid Res. https://doi.org/10.1089/can.2020.0129

4. Aviram J, Pud D, Gershoni T et al (2020) Medical cannabis treatment for chronic pain: outcomes and prediction of response. Eur J Pain. https://doi. org/10.1002/ejp.1675

5. Vachon-Presseau E, Centeno MV, Ren Wetal (2016) The emotional brain as a predictor and amplifier of chronic pain. J Dent Res 95(6):605-612

6. Weizman L, Dayan L, Brill S et al (2018) Cannabis analgesia in chronic neuropathic pain is associated with altered brain connectivity. Neurology 91(14):e1285-e1294

7. Agarwal N, Pacher P, Tegeder I et al (2007) Cannabinoids mediate analgesia largely via peripheral type 1 cannabinoid receptors in nociceptors. Nat Neurosci 10:870-879

8. Fowler CJ (2020) The endocannabinoid system-current implications for drug development JIntern Med.https://doi.org/10.1111/joim.13229

9. Burstein SH (2018) Ajulemic acid: potential treatment for chronic inflammation. Pharmacol Res Perspect 6(2):e00394. https://doi.org/10. 1002/prp2.394

10. Karst M, Salim K, Burstein S et al (2003) Analgesic effect of the synthetic cannabinoid CT-3 on chronic neuropathic pain: a randomized controlled trial. JAMA 290(13):1757-1762

11. Spiera R, Hummers L, Chung L et al (2020) Safety and efficacy of lenabasum in a phase II, randomized, placebo-controlled trial in adults with systemic sclerosis. Arthritis Rheumatol 72(8):1237-1240

12. Aviram J, Lewitus GM, Pud D et al (2021) Specific phytocannabinoid compositions are associated with analgesic response and adverse effects in chronic pain patients treated with medical cannabis. Pharmacol Res. https://doi.org/10.1016/ j.phrs.2021.105651

\section{Anästhesie Update Topaktuell und praxisnah}

12. und 13. November 2021

Umfangreiches Update-Wissen so flexibel wie noch nie: Erstmalig findet das Anästhesie-Intensiv-Update-Seminar als Hybridveranstaltung statt. Bilden Sie sich vor Ort mit Kolleginnen und Kollegen oder bequem von zu Hause aus fort die Zertifizierung ist für beide Formate beantragt.

Das Update-Konzept: Die wichtigsten Neuerungen des vergangenen Jahres aus dem Fachgebiet der Anästhesie und anästhesiologischen Intensivmedizin werden unter der wissenschaftlichen Leitung von Prof. Dr. Grietje Beck (Wiesbaden), Prof. Dr. Winfried Meißner (Jena) und Prof. Dr. Dr. Kai Zacharowski (Frankfurt) kritisch selektiert, analysiert und zusammengefasst. Die Relevanz für den Klinik- und Praxisalltag der Ärztinnen und Ärzte steht dabei im Vordergrund und der ausführlichen Diskussion über den Livechat mit den Referierenden wird viel Raum gegeben.

In diesem Jahr wird neben den Kerngebieten der Anästhesie und anästhesiologischen Intensivmedizin das Thema »Regionalanästhesie« vorgestellt.

Zum Gesamtpaket der Teilnahme gehören neben den umfangreichen Seminarunterlagen der Download aller Vortragspräsentationen und die Vorträge im Nachgang als Video-on-Demand auf der Videoplattform »streamed-up.com«.

Weitere Informationen zu Programm, Referierenden und zur Anmeldung: www. anaesthesie-update.com

Veranstalter: med update $\mathrm{GmbH}$ Hagenauer Straße 53 65203 Wiesbaden 